\title{
Influence of intensity noise in spectrum-sliced WDM systems
}

\author{
Mingshan Zhao, Geert Morthier, Johan Dekoster*, Bart Moeyersoon and Roel \\ Baets \\ Department of Information Technology (INTEC), University of Gent-IMEC, \\ Sint-Pietersnieuwstraat 41, B-9000 Gent, Belgium. \\ *Siemens Atea-ICN OT DH, Atealaan 34, B-2200 Herentals. \\ E-mail: mszhao@intec.rug.ac.be
}

Key words: wavelength division multiplexing, spectral slicing, intensity noise, semiconductor optical amplifier

Abstract: We discuss the influence of intensity noise on the achievable bitrates and transmission distances of spectrum-sliced WDM systems and the reduction of the intensity noise using a saturated SOA. Also the influence of the gainsaturation levels and of the bias currents of the SOA on the noise reduction was studied. For the optimum operation of the saturated SOA, a noise reduction of $17 \mathrm{~dB}$ at low frequencies and $10 \mathrm{~dB}$ at high frequencies was obtained experimentally.

\section{INTRODUCTION}

Spectral-slicing is a WDM technique in which optical filters are used to obtain a spectral slice of light from a broadband source and modulators encode data onto the slice. Spectrum-sliced WDM systems utilising incoherent broadband sources such as LEDs, superluminescent LEDs (SLEDs) and Erbium-doped fibre amplifiers (EDFAs) have advantages of low cost, high wavelength selectivity and temperature stability as compared to conventional DWDM systems. Such systems are strong candidates for future fibre-to-the-home access networks and have potential for costsensitive local area network applications. Spectrum-sliced incoherent light

The original version of this chapter was revised: The copyright line was incorrect. This has been corrected. The Erratum to this chapter is available at DOI: 10.1007/978-0-387-35491-0_28 
however, exhibits a large intensity noise that places limits on the achievable system performances.

In this paper, we present results about the influence of the intensity noise on the achievable bitrates and transmission distances of spectrum-sliced WDM systems and about the reduction of the noise using a saturated semiconductor optical amplifier (SOA). A noise reduction of $17 \mathrm{~dB}$ at low frequencies and $10 \mathrm{~dB}$ at high frequencies was obtained, which is much better than what has so far been published in the literature (10dB at low frequencies) ${ }^{[1]}$.

\section{INFLUENCE OF INTENSITY NOISE ON THE TRANSMISSION PERFORMANCE OF SPECTRUM-SLICED WDM}

The setup used for the transmission experiment using a single spectrum sliced WDM channel is shown in Fig. 1. The amplified spontaneous emission (ASE) from a SLED (SLED 1550MHFD, Opto speed) was spectrally sliced using a fibre Bragg grating (FBG) filter. The slice of light was NRZmodulated using a $\mathrm{LiNbO}_{3}$ external modulator with a $2^{23}-1$ pseudorandombit-sequence (PRBS) pattern, and then detected using a receiver with electrical low pass filters and an oscilloscope (Tektronix 11801B digital sampling oscilloscope).

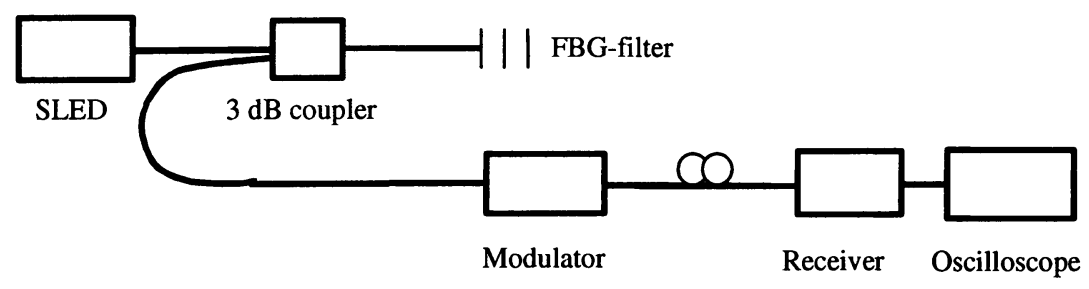

Figure 1. Setup for the fibre link experiment with spectrum-sliced WDM

Fig. 2 shows the eye diagrams of the spectrum-sliced WDM channel for different bitrates and for back to back operation. There is a considerable contribution of intensity noise to the high level of the eye. At low bitrates, e.g. several hundreds of $\mathrm{Mb} / \mathrm{s}$, the eyes are open well, but at high bitrates, e. g. $2.488 \mathrm{~Gb} / \mathrm{s}$, the intensity noise becomes significant and the eyes are almost closed. The eye diagrams for different slice bandwidths are shown in Fig. 3. Larger slicewidth results in a higher signal to noise ratio and thus a better eye diagram. These results are consistent with theoretical analysis. With the 
assumption of a Gaussian probability density function, the signal to noise ratio (SNR) of a spectral slice is given by

$$
S N R=\sqrt{\frac{B_{o}}{2 B_{e}}}=\sqrt{\frac{2 B_{o}}{3 B}}
$$

when intensity noise dominates (that means, thermal noise and shot noise can be neglected), where $B_{e}(=0.75 B)$ is the electrical bandwidth of the receiver, $B_{o}$ the optical bandwidth of the spectral slice, and $B$ the data bitrate.

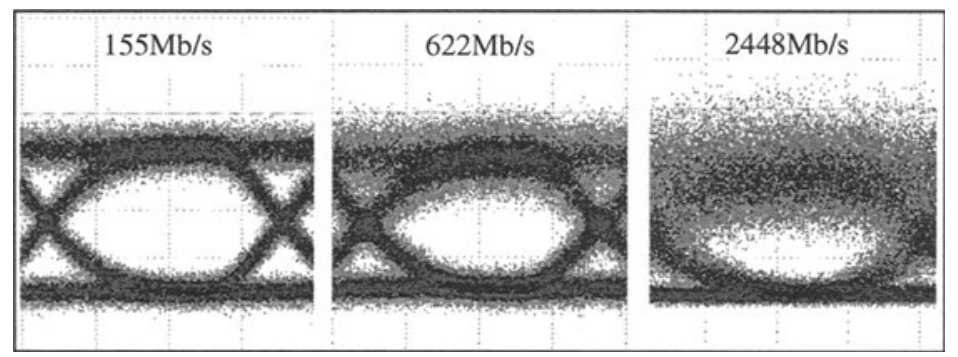

Figure 2. Eye diagrams for different bitrates at back to back operation. Slicewidth: $0.5 \mathrm{~nm}$

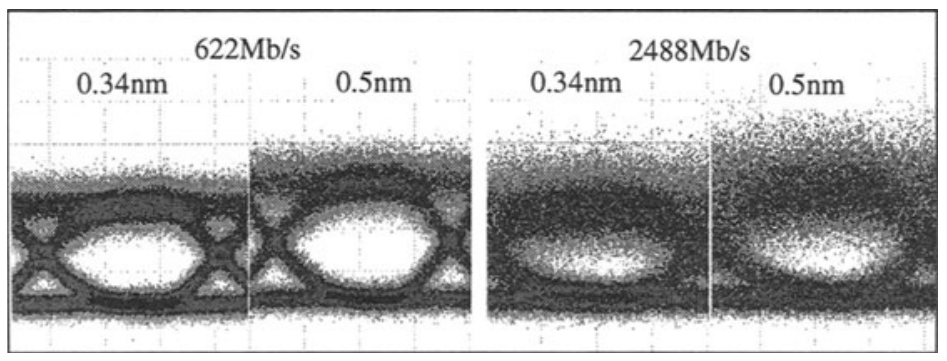

Figure 3. Eye diagrams for different slicewidths and different bitrates. Back to back operation

Equation 1 shows that the signal to intensity noise ratio of a spectral slice is proportional to the square root of the slicewidth $B_{o}$, but inversely proportional to the square root of the data bitrate $B$. For a given slicewidth, higher bitrate results in a lower SNR. A lower bitrate results in a higher SNR and thus a larger eye height. For a given bitrate $B$, the SNR can be increased by increasing the slicewidth. However, a larger slicewidth also leads to larger dispersion induced inter-symbol interference, which seriously degrades the 
system performance. So, intensity noise places limits to the bitrate and transmission distance in spectrum-sliced WDM. With PTDS (Photonic Transmission Design Suite) from Virtual Photonics Incorporated ${ }^{[2]}$, simulations of the transmission of a single spectrum-sliced WDM channel were performed. The results show that, as can be seen in Fig. 4 and 5, for a bitrate of $625 \mathrm{Mb} / \mathrm{s}$, transmission over $100 \mathrm{~km}$ with a BER of $10^{-9}$ can be achieved, for a slicewidth of $0.8 \mathrm{~nm}$. But for a bitrate of $2.5 \mathrm{~Gb} / \mathrm{s}$, less than $10 \mathrm{~km}$ transmission with a BER of $10^{-9}$ can be achieved, for a slicewidth of $1.4 \mathrm{~nm}$.

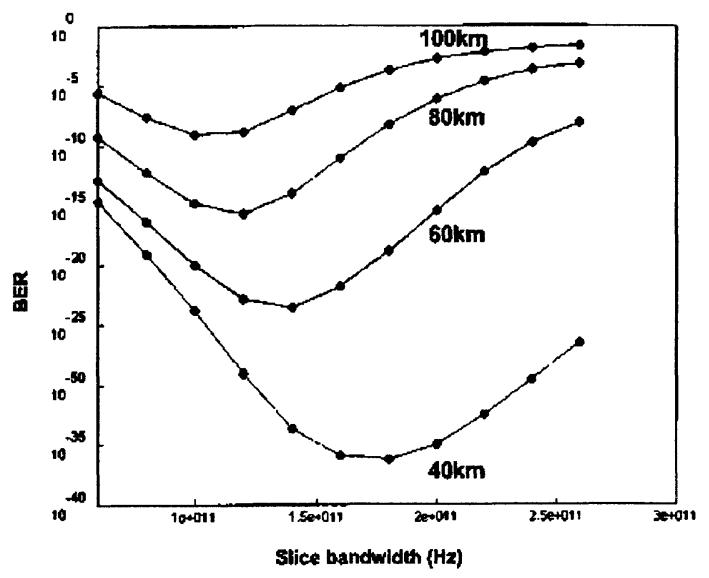

Figure 4. BER vs. slicewidth for different lengths of fibre links at a bitrate of $625 \mathrm{Mb} / \mathrm{s}$.

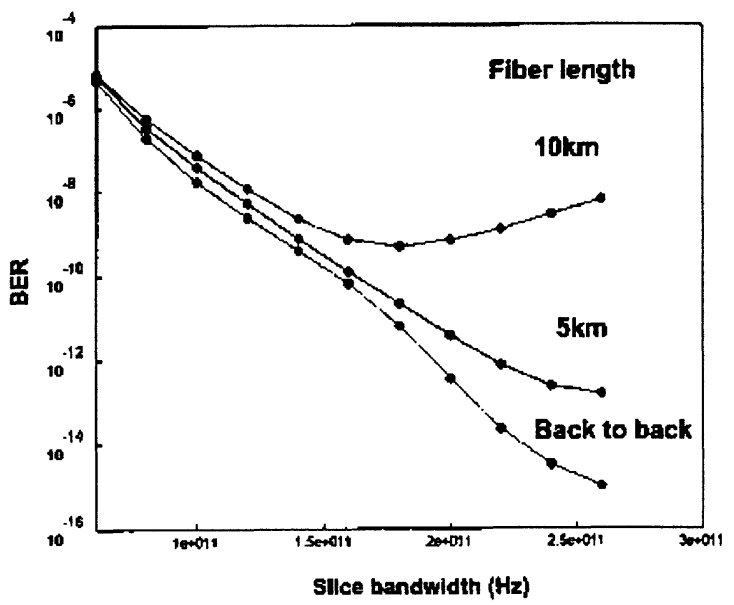

Figure 5. BER vs. slicewidth for different lengths of fibre links at a bitrate of $2.5 \mathrm{~Gb} / \mathrm{s}$. 


\section{REDUCTION OF INTENSITY NOISE IN SPECTRUM-SLICED WDM USING A SATURATED SOA}

Both the experimental and the simulation results given in the above section have shown that large intensity noise in spectrum-sliced WDM strongly limits transmission bitrate and distance. In order to break the limitations and improve the performance of spectrum-sliced WDM, the noise property of the spectral slice must be modified. A saturated SOA exhibits a suppressed intensity noise for narrow band detection ${ }^{[3]}$. Techniques for reducing the intensity noise using a saturated SOA have recently been proposed and experimentally demonstrated ${ }^{[1,4]}$. We have further investigated the possibility of intensity noise reduction using a saturated SOA. The influence of the gain-saturation level and the bias current of the SOA on the noise reduction has been investigated experimentally, and the optimum condition for a high noise suppression ratio has been derived. In contrast to previous experiments we have used a Gain-Clamped SOA (GC-SOA).

Our experimental setup for intensity noise reduction is shown in Fig. 6. The ASE from a SLED was spectrally sliced using a $0.5 \mathrm{~nm}$-bandwidth FBG filter. The spectrum-sliced incoherent light centred at $1550.8 \mathrm{~nm}$ was amplified using an EDFA and then injected into a gain saturated GC-SOA. The output of the GC-SOA was modulated using a $\mathrm{LiNbO}_{3}$ external modulator. To remove the ASE from the amplifiers and the lasing-mode from the GC-SOA, a 1.2-nm bandwidth filter was used before the modulator. With a lightwave signal analyser (HP71400), a digital sampling oscilloscope (Tektronix) and an Error Detector (HP70842A), relative intensity noise, eye diagrams and BERs with and without GC-SOA were measured, respectively.

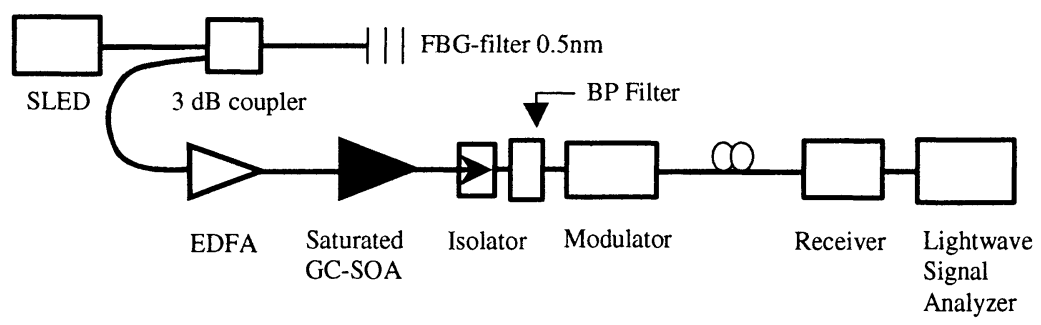

Figure 6. Experimental setup for intensity noise reduction

Figs. 7 and 8 show the measured relative intensity noise spectra of spectrum-sliced incoherent light and the noise suppression ratios, respectively. The noise level of the input was significantly suppressed over a bandwidth of several $\mathrm{GHz}$. The amount of the noise reduction is proportional 
to the gain compression (difference between a large signal gain and a small signal gain) and bias current of the SOA but depending on the frequency. At low frequency, a noise reduction of $17 \mathrm{~dB}$ was obtained with a high bias current of the GC-SOA. At high frequency, e. g. $2.5 \mathrm{GHz}, 10 \mathrm{~dB}$ reduction of the intensity noise can be reached.

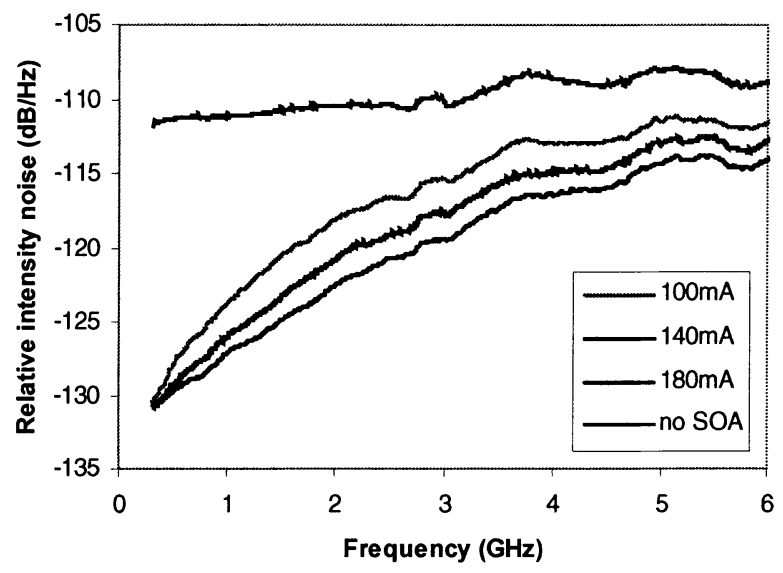

Figure 7. Measured relative intensity noise without and with a gain-saturated GC-SOA at different bias currents

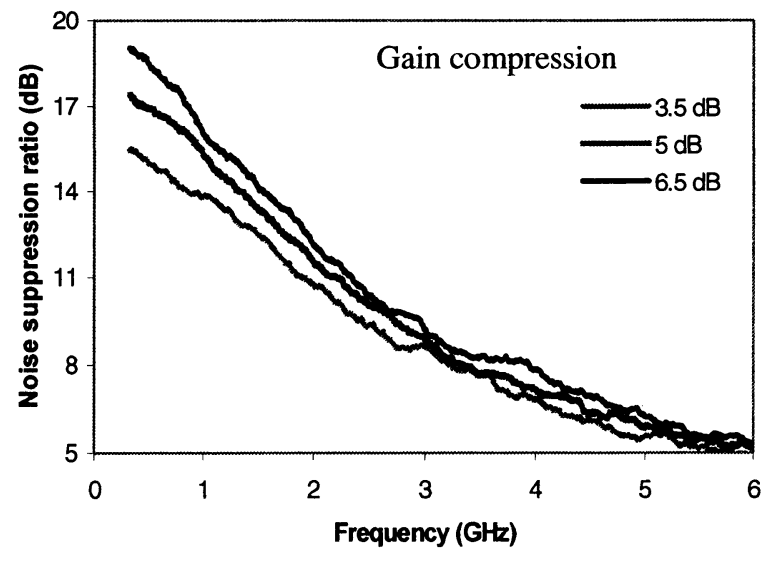

Figure 8. Noise suppression ratio for different gain-saturation levels

These points are shown more clearly in Fig. 9. At low frequency, e. g. $622 \mathrm{MHz}$, the noise suppression ratio increases both with increasing bias current and with increasing gain-saturation level of the GC-SOA, but the 


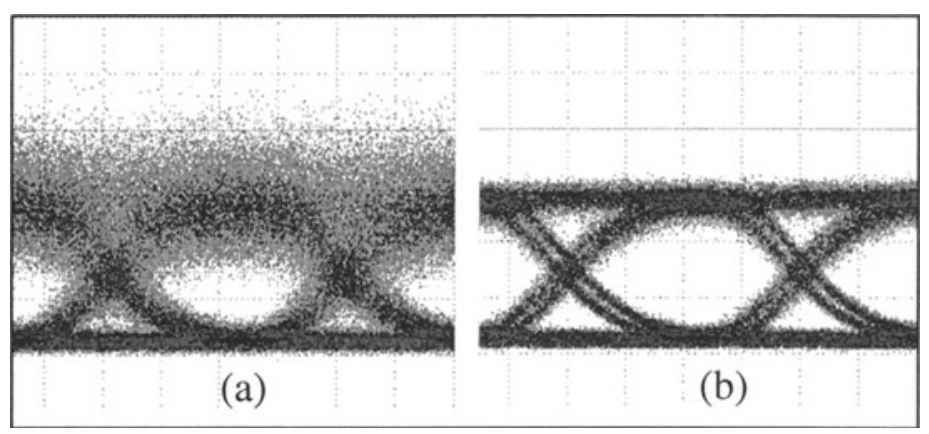

Figure 10. Eye diagrams for back to back operation for a data bitrate of $2.488 \mathrm{~Gb} / \mathrm{s}\left(2^{23}-1\right.$ PRBS): (a) without and (b) with GC-SOA

Fig. 10 shows the eye diagrams for $2.488 \mathrm{~Gb} / \mathrm{s}$ back to back operation. With the GC-SOA, the intensity noise is suppressed dramatically. The bias current of the GC-SOA is $180 \mathrm{~mA}$, and the gain compression is about $6.5 \mathrm{~dB}$.

We also measured the BER for $622 \mathrm{Mb} / \mathrm{s}$ back to back operation. The results show that without GC-SOA the BER is rather high and there is a floor at around $1 \times 10^{-6}$. However, with GC-SOA the bit error rate is improved by several orders of magnitude and the error floor is removed. When the received power is larger than $-18 \mathrm{dBm}$, the transmission is error free. More experiments about the influence of gain saturation level and bias current on the BER are on going.

\section{CONCLUSION}

We have presented the influence of the intensity noise on the achievable bitrates and transmission distances of spectrum-sliced WDM systems and the reduction of the intensity noise using a saturated SOA. The influence of the gain-saturation level and the bias current of the SOA on the noise reduction has also been studied. For the optimum operation of the SOA, a noise reduction of $17 \mathrm{~dB}$ at low frequency and $10 \mathrm{~dB}$ at high frequency was obtained experimentally.

\section{ACKNOWLEDGEMENTS}

This work has been supported by the Flemish Government-IWT. 
dependence on bias current is less strong, particularly in the high bias current region. At high frequency, e. g. $2.5 \mathrm{GHz}$, the noise suppression ratio increases with increasing bias current, but it is not effective to improve the noise suppression by increasing the gain-saturation level. These experimental results above are consistent with the theoretical analyses on the noise characteristics of a saturated $\mathrm{SOA}^{[3]}$, which show that the $3 \mathrm{~dB}$ bandwidth of noise suppression for a given input power is proportional to the intensity of the output and thus the bias current of the saturated SOA. So, in practical applications we can improve the noise suppression by increasing the gainsaturation level of the SOA at low bitrate and the bias current at high bitrate.
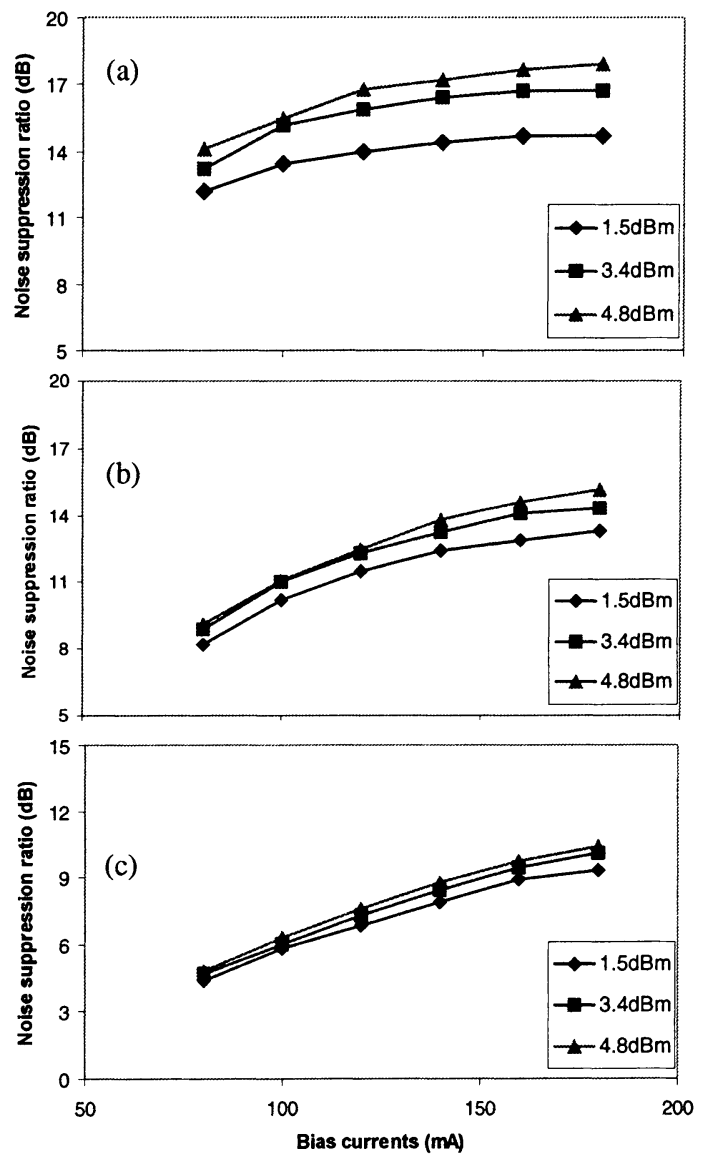

Figure 9. Noise suppression ratios vs. bias currents of the GC-SOA at different frequencies and input powers, (a) $622 \mathrm{MHz}$, (b) $1.25 \mathrm{GHz}$, and (c) $2.5 \mathrm{GHz}$ 
References:

[1] T. Yamatoya, F. Koyama and K. Iga, "Noise suppression and intensity modulation using gain-saturated semiconductor optical amplifier", Technical Digest, Integrated Photonics Research 2000, Canada, July, 2000. pp. 72-74.

[2] PTDS was supplied by Virtual Photonics Incorporated: www.virtualphotonics.com.

[3] M. Shtaif and G. Eisenstein, "Noise characteristics of nonlinear semiconductor optical amplifiers in the Gaussian limit", IEEE J. Quantum Electron., Vol. 32, pp. 1801-1809, 1996.

[4] S. J. Kim, J. H. Han, J. S. Lee and C. S. Park, "Intensity noise suppression in spectrumsliced incoherent light communication systems using a gain-saturated SOA", Photonics Technology Letters, Vol. 11, No. 8, 1999, P 1042-1044. 